Pacific Journal of Mathematic 


\section{SUBORDINATION AND EXTREME-POINT THEORY}

\section{J. Hallenbeck AND T. H. MacGregor}

This paper examines the set of extreme points of the convex hull of families of analytic functions defined through subordination. The set of extreme points is determined for the class of functions each of which is subordinate to some starlike, univalent mapping of the open unit disk. This set is also determined for the family defined by subordination to some convex mapping, and a partial determination is obtained for subordination to some close-to-convex mapping. This information is used to solve extremal problems over such families. Results are also presented about the extreme points for the functions which are subordinate to a given analytic function $F$. For example, if $f(z)=F(x z)$ and $|x|=1$ then $f$ is an extreme point. If $F \in H^{p}, 1<p<\infty$, and $\phi$ is an inner function with $\phi(0)=0$, then $F(\phi)$ is an extreme point.

We recall the definition of subordination between two functions, say $f$ and $F$, analytic in $\Delta=\{z:|z|<1\}$. This means that $f(0)=F(0)$ and there is an analytic function $\phi$ so that $\phi(0)=0,|\phi(z)|<1$ and $f(z)=F(\phi(z))(z \in \Delta)$. This relation shall be denoted by $f \prec F$. If $F$ is univalent in $\Delta$ the subordination is equivalent to $f(0)=F(0)$ and $f(\Delta) \subset F(\Delta)$. (See [8, p. 421-] and [13, p. 226-] for some basic results about subordination.)

The classical illustration of our interest is the family $\mathscr{P}$ of functions with a positive real part. Recall that $f \in \mathscr{P}$ if $f$ is analytic, $f(0)=1$ and $\operatorname{Re} f(z)>0(z \in \Delta)$. The family $\mathscr{P}$ consists of the functions subordinate to $F(z)=(1+z) /(1-z)$ in $\Delta$. Herglotz's formula $f(z)=\int_{|x|=1}(1+x z) /(1-x z) d \mu(x)$ gives a representation of the functions in $\mathscr{P}$, where $\mu$ varies over the probability measures on the unit circle. Since the mapping $\mu \rightarrow f$ is one-to-one this formula also implies that the extreme points of $\mathscr{P}$ are exactly the functions $f(z)=$ $(1+x z) /(1-x z),|x|=1[14$, p. 30].

We shall let $\mathscr{A}$ denote the set of functions analytic in $\Delta$. This set is a locally convex linear topological space with respect to the topology of uniform convergence on compact subsets of $\Delta[19, \mathrm{p} .150]$. Thus, the ideas of extreme points, convex hulls and other terms are meaningful, and the general results about such spaces are applicable. (We refer to [5, Chapter 5] for these considerations.) We shall use the notaion $\mathscr{S C}_{\mathscr{F}}$ to denote the closed convex hull of the set $\mathscr{F}$ and ES. $\mathscr{F}$ to denote the extreme points of $\mathscr{S C}_{\mathscr{F}}$.

The functions analytic in $\Delta$ and with a range contained in a given half-plane can be represented by a formula analogous to the Herglotz 
formula by a simple appeal to that formula. With the normalization $f(0)=1$ such a family consists of the functions subordinate to $F(z)=$ $(1+c z) /(1-z)$ for a suitable number $c$. The representation formula is $f(z)=\int_{|x|=1}(1+c x z) /(1-x z) d \mu(x)$ and the functions $(1+c x z) /(1-x z)$, $|x|=1$, are the extreme points.

A more recent example of this kind of relation was obtained in [2, see Theorem 5]. The result proved there implies the following assertion: If $\mu$ is a probability measure on $|x|=1$ then there is another probability measure $\nu$ on $|x|=1$ such that

$$
\left\{\int_{|x|=1} \frac{1}{1-x z} d \mu(x)\right\}^{p}=\int_{|x|=1} \frac{1}{(1-x z)^{p}} d \nu(x) \quad(z \in \Delta)
$$

$(p=1,2,3, \cdots)$. The functions given by the left-hand side of equation (1) are precisely the analytic functions subordinate to $F(z)=$ $1 /(1-z)^{p}$ in $\Delta$. The functions defined by the right-hand side of the equation form a closed convex set, and since the kernel functions $f(z)=1 /(1-x z)^{p}(|x|=1)$ are subordinate to $F(z)$ we may make the following conclusions. The closed convex hull of the functions subordinate to $F(z)=1 /(1-z)^{p}$ is given by the set of functions $\int_{|x|=1} 1 /(1-x z)^{p} d \mu(x)$, where $\mu$ varies over the probability measures on $|x|=1$, and the extreme points of this hull are the functions $f(z)=1 /(1-x z)^{p},|x|=1(p=1,2,3, \cdots)$.

A very recent result of D. Brannan, J. Clunie, and W. Kirwan in [1] implies that the conclusions described above more generally hold if $p \geqq 1$. This corresponds to the special case $c=0$ of the following assertion.

Theorem A. (Brannan, Clunie, Kirwan) Let $c$ be a complex number, $|c| \leqq 1$, and let $\mathscr{F}$ denote the family of functions analytic in $\Delta$ and subordinate to $F(z)=((1+c z) /(1-z))^{p}$, where $p \geqq 1$. Then $\mathfrak{S} \mathscr{F}$ consists of the functions $f(z)=\int_{|x|=1}((1+c x z) /(1-x z))^{p} d \mu(x)$, where $\mu$ varies over the probability measures on $|x|=1$. Moreover, अ5 $\mathscr{F}$ are the functions $f(z)=[(1+c x z) /(1-x z)]^{p},|x|=1$.

Theorem A implies that if $p \geqq 1,|c| \leqq 1$ and if $\mu$ is a probability measure on $|x|=1$ then there is another probability measure $\nu$ on $|x|=1$ such that

$$
\left\{\int_{|x|=1} \frac{1+c x z}{1-x z} d \mu(x)\right\}^{p}=\int_{|x|=1}\left(\frac{1+c x z}{1-x z}\right)^{p} d \nu(x) \quad(z \in \Delta) .
$$

Conversely, this generalization of the result concerning equation (1) 
implies Theorem A, as indicated by the argument following equation (1).

Our main interest in Theorem $\mathrm{A}$ is in the special case $p=2$. We use Theorem A with $p=2$ and $c=1$ to determine the convex hull and the extreme points of the functions subordinate to some starlike mapping. (In fact, the present authors proved Theorem A in this special case by a different method and independently of Brannan, Clunie, and Kirwan.) To be more precise, let $S$ denote the subset of $\mathscr{A}$ of functions univalent in $\Delta$ so that $f(0)=0$ and $f^{\prime}(0)=$ 1. Also, let $S t$ denote the subset of $S$ so that $f \in S t$ if $f(\Delta)$ is starlike with respect to 0 . Let $S t^{*}=\{f: f \prec g$ for some $g$ in $S t\}$. (In general if $\mathscr{F} \subset \mathscr{A}$ we let $\mathscr{F}^{*}$ denote the set $\{f: f \prec g$ for some $g$ in $\mathscr{F}\}$.) We find $\mathfrak{S}_{2} S t^{*}$ and ${ }_{5} S S t^{*}$. The argument depends upon knowing (5) S S St, as determined in [2, Theorem 3], some observations made in [12] and the special case of Theorem A.

In the same way, Theorem A with $p=2$ and $|c|=1$ is used to discuss the convex hull and extreme points of functions subordinate to some close-to-convex function. We recall that the family of closeto-convex functions, which we denote by $C$, consists of the functions $f$ analytic in $\Delta$, so that $f(0)=0, f^{\prime}(0)=1$ and for which there exist a complex number $a$ and a function $g$ so that $\operatorname{Re}\left\{z f^{\prime}(z) / g(z)\right\}>0(z \in \Delta)$ and $a g \in S t$. This class was introduced by W. Kaplan in [10] and we note that $S t \subset C \subset S$.

Let $K$ denote the subset of $S$ of functions $f$ for which $f(\Delta)$ is convex. We determine $\mathfrak{S E} K^{*}$ and ${ }_{\mathfrak{S} S \mathcal{E}} K^{*}$. We also give applications of our results to the solution of extremal problems and indicate other families for which these considerations are useful.

The last part of this paper concerns the following general question. Suppose that $F \in \mathscr{A}$ and $\mathscr{F}$ denotes the family of functions subordinate to $F$ in $\Delta$. What, in general, can be said about $5 F_{\mathcal{C}} \mathscr{F}$ ?

We first show that the functions $f(z)=F(x z),|x|=1$, always belong to $55_{\mathcal{C}} \mathscr{F}$. That these functions may be the only functions in (55 $\mathscr{F}$ is well illustrated by the previous examples. The quite opposite situation is represented by the diversity of the set of extreme points of the functions in $\mathscr{A}$ that satisfy $|f(z)| \leqq 1(z \in \Delta)$ [see 9, p. 138]. (This is in the context of our discussion where, say, $F(z)=z$.) In this direction, we show that if $F \in H^{p}$ (the Hardy class), where $1<$ $p<\infty$ and if $\phi$ is an inner function and $\phi(0)=0$, then $F(\phi)$ is an extreme function for the class.

2. The extreme points and convex hull of $K^{*}, S t^{*}$, and $C^{*}$.

THEOREM 1. Let $K$ denote the subset of $S$ of convex mappings 
and let $K^{*}=\{f: f<g$ for some $g$ in $K\}$ and let $X=U \times U$ where $U=\{z:|z|=1\}$. Then $\mathfrak{S} K^{*}$ consists of the functions given by

$$
f(z)=\int_{X} \frac{x z}{1-y z} d \mu(x, y) \quad(z \in \Delta)
$$

where $\mu$ varies over the probability measures on $X$. Also, (5Sב $K^{*}=$ $\{f: f(z)=x z /(1-y z),|x|=|y|=1\}$.

Proof. Suppose that $f \in \mathbb{F} \mathcal{S}_{\mathcal{C}} K^{*}$. The argument given in [12, see p. 366] implies that $f \prec g$ for some function $g$ in $55 \mathcal{F} K$. Since $5 S_{\mathcal{C}} K$ is the set of functions $g(z)=z /(1-x z),|x|=1$ [see 2, Theorem 2] we conclude that $f$ is subordinate to $g$ where $g(z)=z /(1-x z)$ and $|x|=1$.

Let $K(x)=\{f: f \prec g, g(z)=z /(1-x z)\}$ where $x$ is fixed, $|x|=1$. Then, because $z /(1-x z)=-1 / x+1 / x 1 /(1-x z)$, we see that $f(z)=$ $-1 / x+1 / x h(z)$ where $h \prec k$ and $k(z)=1 /(1-x z)$. The collection $\{h\}$ consists of the function subordinate to $w=1 /(1-z)$ and so we con-

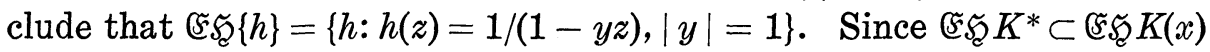
for all $x(|x|=1)$ it follows that $f \in \mathscr{S} S_{\mathcal{C}} K(x)$ and thus $f(z)=-1 / x+$ $1 / x 1 /(1-y z)$ for some $y,|y|=1$. This is the same as $f(z)=$ $y z / x(1-y z)$, and so (with a change of letters) we conclude that FSS $K^{*} \subset E=\{f: f(z)=x z /(1-y z),|x|=|y|=1\}$.

We next show that $E \subset \lessdot 5 \mathfrak{S} K^{*}$. Because $E \subset K^{*}$ this will follow if we show that each function in $E$ uniquely maximizes a real-valued continuous linear functional over $E$. Let $J(f)=\alpha f^{\prime}(0)+\beta f^{\prime \prime}(0) / 2$ where $\alpha$ and $\beta$ are two complex numbers with $|\alpha|=|\beta|=1$. Then, if $f \in E, \quad \operatorname{Re} J(f)=\operatorname{Re}[\alpha x+\beta x y] \leqq|\alpha x+\beta x y| \leqq 2$. The equality $\operatorname{Re} J(f)=2$ determines that $x=1 / \alpha$ and $y=\alpha / \beta$. Thus, a unique function in $E$ maximizes $\operatorname{Re} J(f)$. By varying $\alpha$ and $\beta$ this gets all possible pairs $x, y$. This completes the proof that $5 S_{\mathcal{C}} K^{*}=E$.

The conclusion that $\mathfrak{S} K^{*}$ is given by the functions represented by equation (3) follows from the Krein-Milman theorem [5, p. 440]. This uses the fact that the collection of functions $\mathfrak{S} K^{*}$ is a compact convex set and that $\mathfrak{F S S}_{\mathcal{S}} K^{*}=\{f: f(z)=x z /(1-y z),|x|=|y|=1\}$. We also appeal to [2, Theorem 1].

THEOREM 2. Let $S t^{*}=\{f: f \prec g$ for some $g$ in $S t\}$, let $U=$ $\{x:|x|=1\}$ and let $X=U \times U$. Then $\mathfrak{S C S t}^{*}$ consists of the functions represented by

$$
f(z)=\int_{X} \frac{y z}{(1-x z)^{2}} d \mu(x, y)
$$

where $\mu$ varies over the probability measures on $X$. Also, 


$$
\mathfrak{F S S C}_{\mathcal{C}} S t^{*}=\left\{f: f(z)=\frac{y z}{(1-x z)^{2}},|x|=|y|=1\right\} \text {. }
$$

Proof. Suppose that $f \in$ (5SSSt. Then, by the argument given

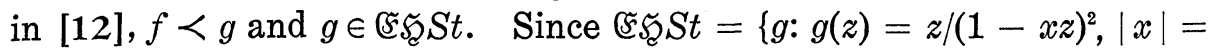
1) [see 2, Theorem 3] we conclude that $f \prec g$ where $g(z)=z /(1-x z)^{2}$ and $|x|=1$.

Let $\mathscr{S}(x)$ denote the set of functions subordinate to $F(x, z)=$ $z /(1-x z)^{2},|x|=1$. The identity $F(x, z)=1 / 4 x\left[((1+x z) /(1-x z))^{2}-1\right]$ shows that the linear $\operatorname{map} f=1 / 4 x(g-1)$ exhibits a one-to-one correspondence between the family $\mathscr{S}(x)$ and the class of functions $\mathscr{F}$ subordinate to the function $F(z)=[(1+z) /(1-z)]^{2}$. Therefore, $\mathscr{S} \mathscr{S}(x)=1 / 4 x(\mathscr{S} \mathscr{F}-1)$ and $\mathbb{F} \mathscr{S} \mathscr{S}(x)=1 / 4 x($ FS $\mathscr{F}-1)$. The case $c=1, p=2$ of Theorem A shows that $\mathbb{F} \mathscr{S} \mathscr{F}$ consists of the functions $w=((1+y z) /(1-y z))^{2},|y|=1$. Since ${ }_{5} S_{S} S t^{*} \subset$ (5SS $\mathscr{S}(x)$ we conclude that $f(z)=1 / 4 x\left[((1+y z) /(1-y z))^{2}-1\right]$. In a slightly different notion, if we let $\mathscr{G}=\left\{f: f(z)=y z /(1-x z)^{2},|x|=|y|=1\right\}$, this proves that (FSS $S t^{*} \subset \mathscr{G}$.

We next show that $\mathscr{G} \subset$ $5 S_{C} S t^{*}$. To do this we prove that to each function $f_{0}$ in $\mathscr{G}$ there a continuous linear functional $J$ on $\mathscr{A}$ such that $f=f_{0}$ is the only function in $\mathscr{G}$ for which $\operatorname{Re} J(f)=$ $\max \{\operatorname{Re} J(g): g \in \mathscr{G}\}$. Specifically, let $\alpha$ and $\beta$ be complex numbers so that $|\alpha|=|\beta|=1$ and let $J(g)=\alpha g^{\prime}(0)+\beta g^{\prime \prime}(0)$. Then $\operatorname{Re} J(g)=$ $\operatorname{Re}[\alpha y+4 \beta y x] \leqq|\alpha y|+4|\beta y x|=5$. The maximum value 5 is only achieved if $y=1 / \alpha$ and $x=\alpha / 4 \beta$. Letting $\alpha$ and $\beta$ vary we get all possible pairs $x, y$ associated with the unique function in $\mathscr{G}$ with $\operatorname{Re} J(g)=5$. This completes the proof of the determination of ${ }_{5} S_{C} S t^{*}$.

The assertion about $\mathfrak{S C}_{\mathcal{S}} t^{*}$ given through equation (10) is a consequence of the Krein-Milman theorem and [2, Theorem 1], since $\mathfrak{S C S}_{\text {St }}$. is compact and convex and the set of kernel functions for all possible choices of $x$ and $y$ is precisely equal to the set ${ }_{5} S S t^{*}$.

THEOREM 3. Let $C$ denote the subset of $S$ of close-to-convex functions and let $C^{*}=\{f: f \prec g$ for some $g$ in $C\}$. Let $X=U \times U \times U$ where $U=\{z:|z|=1\}$. Then $\mathfrak{S C}^{*}$ consists of all functions

$$
f(z)=\int_{X} w \frac{z+[w(x+y) / 2] z^{2}}{(1+w x z)^{2}} d \mu(x, y, w)
$$

where $\mu$ varies over the probability measures on $X$. Also, 


$$
\text { FSS } C^{*} \subset\left\{f: f(z)=w \frac{z+[w(x+y) / 2] z^{2}}{(1+w x z)^{2}},|x|=|y|=|w|=1, x \neq y\right\} .
$$

Proof. This is similar to the proofs of Theorems 1 and 2. Sup-

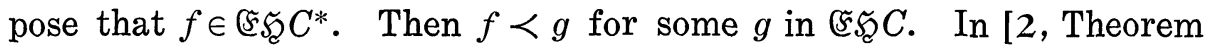
6] $\left[5 S_{C} C\right.$ was completely determined as the set of functions

$$
g(z)=\frac{z-(x+y) / 2 z^{2}}{(1-y z)^{2}},|x|=|y|=1, \quad x \neq y
$$

and so $f \prec g$ for such a function $g$. We may write

$$
g(z)=\frac{1}{2(y-x)}\left[\left(\frac{1-x z}{1-y z}\right)^{2}-1\right]
$$

and therefore,

(4) $f(z)=\frac{1}{2(y-x)}[h(z)-1]$ where $h \prec k$ and $k(z)=\left(\frac{1-x z}{1-y z}\right)^{2}$.

The collection $\{h\}$ is the same as the set of functions subordinate to $[(1-u z) /(1-z)]^{2}$ where $u=x / y \neq 1,|u|=1$.

According to Theorem A with $c=-u$ and $p=2$ the extreme points of the hull of $\{h\}$ are the functions $[(1-u v z) /(1-v z)]^{2}$ where $|v|=1$. The linear relation (4) also holds between the two sets of extreme points of the hulls of $\{f\}$ and $\{h\}$ and so an extreme $f$ must have the form

$$
\begin{aligned}
f(z) & =\frac{1}{2(y-x)}\left[\left(\frac{1-(y / x) v z}{1-v z}\right)^{2}-1\right] \\
& =-\frac{v}{x} \frac{z-(v / 2 x)(x+y) z^{2}}{(1-v z)^{2}} .
\end{aligned}
$$

Therefore,

$$
\begin{gathered}
\mathfrak{F S S C}_{\mathfrak{C}} C^{*} \subset E=\left\{f: f(z)=w \frac{z+w[(x+y) / 2] z^{2}}{(1+w x z)^{2}},\right. \\
|x|=|y|=|w|=1, x \neq y\}
\end{gathered}
$$

Our assertion about $\mathfrak{S C}^{*}$ follows in the usual way by appealing to the Krein-Milman theorem and the fact that $5 S_{\mathcal{S}} C^{*} \subset E$. We are unable to resolve the question of whether $\mathbb{F} S_{\mathcal{C}} C^{*}=E$. In any case, the inclusion ${ }^{5} S_{C} C^{*} \subset E$ suffices for most applications.

3. Applications to extremal problems and other classes of functions. 
THEOREM 4. Let $R(\alpha)$ denote the set of analytic functions $f$ so that $f(0)=0, f^{\prime}(0)=1$ and $\operatorname{Re} \sqrt{f(z) / z}>\alpha(z \in \Delta)(0 \leqq \alpha<1)$. Let $X$ denote the unit circle, $P$ the set of probability measures on $X$, and

$$
f_{\mu}(z)=\int_{X} z\left(\frac{1+(1-2 \alpha) x z}{1-x z}\right)^{2} d \mu(x)
$$

Then

$$
\begin{aligned}
\mathfrak{S} R(\alpha) & =\left\{f_{\mu}: \mu \in P\right\} \text { and } \mathfrak{F} \mathfrak{S}_{\mathcal{C}} R(\alpha) \\
& =\left\{f: f(z)=z\left(\frac{1+(1-2 \alpha) x z}{1-x z}\right)^{2}, x \in X\right\}
\end{aligned}
$$

Proof. The result follows by the usual argument by applying Theorem A where $c=1-2 \alpha$ and $p=2$.

We remark that $R(0) \supsetneq T$ where $T$ is the set of typically real functions introduced by Rogosinski [16]. This fact is probably known but we point out here that if $f \in T$ then $f(z)=\left[z /\left(1-z^{2}\right)\right] p(z)$ where $p \in \mathscr{P}$ (and $p$ is real on $(-1,1)$ ) and the general fact that if $\operatorname{Re} w_{1}>$ 0 and $\operatorname{Re} w_{2}>0$ then $\operatorname{Re} \sqrt{w_{1} w_{2}}>0$. We also note that the class $S \cap R(1 / 2)$ was introduced by O. Dvorak in [6] and most recently discussed by P. Duren and G. Schober in [4]. We recall that $S \cap$ $R(1 / 2) \supsetneqq S t$ and yet as is clear from a result in [2, p. 106] and the above theorem where $\alpha=1 / 2$, that $\mathfrak{S} R(1 / 2)=\mathfrak{S C}_{\mathcal{C}}(S \cap R(1 / 2))=\mathfrak{S C}_{\mathcal{C}} S t$. Note however that $\mathfrak{S C} R(0) \neq \mathfrak{S} T=T$.

It is also clear that if one defines the class $R_{p}(\alpha)$ through the condition $\operatorname{Re} \sqrt[p]{f(z) / z}>\alpha$ and with the above normalizations then if $p \geqq 1$ again by applying Theorem $\mathrm{A}$ we find that

$$
\mathfrak{S}_{\mathcal{C}} R_{p}(\alpha)=\left\{f_{\mu}(z): \mu \in P\right\} \text { where } f_{\mu}(z)=\int_{X} z\left(\frac{1+(1-2 \alpha) x z}{1-x z}\right)^{p} d \mu(x)
$$

and $X$ and $P$ are as defined above.

Theorem 5. Let $\mathscr{F}=\{f: f \prec F\}$ where $F(z)=(1-z)^{-p}$. If $f \in \mathscr{F}, p \geqq 1$ and

$$
f(z)=\sum_{n=0}^{\infty} a_{n} z^{n}, \text { then }\left|a_{n}\right| \leqq \frac{p(p+1) \cdots(p+n-1)}{n !}(n=1,2, \cdots) .
$$

Proof. It suffices to examine the set $\mathbb{F} \mathscr{S} \mathscr{F}$. By applying Theorem A with $c=0$ one finds that

$$
\text { F } \mathfrak{S}_{\mathfrak{F}}=\left\{f: f(z)=(1-x z)^{-p},|x|=1\right\} \text {. }
$$


The result follows since $F(z)=1+\sum_{n=1}^{\infty} A_{n} z^{n}$ with

$$
A_{n}=\frac{p(p+1) \cdots(p+n-1)}{n !} .
$$

We remark that when $p \geqq 2$ this theorem is obtainable by a result of Rogosinski $[17, \mathrm{p} .64]$ since the numbers $A_{k}(1 \leqq k \leqq n)$ are nondecreasing and convex. That sequence is not convex if $1<p<$ 2. When $p \leqq 1$ the sharp coefficient estimate is $\left|a_{n}\right| \leqq p(n=1,2, \cdots)$.

We recall the result of Rogosinski [17, p. 72] that if $f<g$ and $g \in S t$ then $\left|a_{n}\right| \leqq n(n=1,2, \cdots)$ where $f(z)=\sum_{n=1}^{\infty} a_{n} z^{n}$. An application of Theorem 4 with $\alpha=1 / 2$ and the kind of argument made in the proof of Theorem 2 shows that $₹ 5 \mathcal{C} R^{*}(1 / 2)=$ ES S S $t^{*}$. This implies a generalization of Rogosinski's result; namely, if $f \prec F, F \in R(1 / 2)$ and $f(z)=\sum_{n=1}^{\infty} a_{n} z^{n}$ then $\left|a_{n}\right| \leqq n(n=1,2, \cdots)$. For, it suffices to consider the functions in $5 S_{\mathcal{C}} R^{*}(1 / 2)$, and the $n$th coefficient of a function $f(z)=y z /(1-x z)^{2},|x|=|y|=1$, always has modulus $n$.

In [15] Robertson generalized the result of Rogosinsk for the coefficients of a function in $C^{*}$. By making use of Theorem 3 we can obtain a simple proof of this result. It suffices to consider the function in $\Im_{5} C^{*}$. Hence we need only show that the $n$th coefficient of the functions

$$
f(z)=w \frac{z+[w(x+y) / 2] z^{2}}{(1+w y z)^{2}}, \quad|w|=|x|=|y|=1,
$$

has modulus bounded by $n$. This is an easy calculation; namely

$$
a_{n}=\frac{1}{2}\left[(n+1) y^{n-1}-(n-1) x y^{n-2}\right](-w)^{n},
$$

and so

$$
\left|a_{n}\right| \leqq 1 / 2[n+1+n-1]=n .
$$

Our next applications deal with the $L^{p}$ means of a function $f$ which is subordinate to a function $g$ belonging to some compact subfamily $\mathscr{F}$ of $S$. To each function $f$ analytic in $\Delta$ we let

$$
J(f)=\frac{1}{2 \pi} \int_{0}^{2 \pi}\left|f\left(r e^{i \theta}\right)\right|^{p} d \theta \text {, where } 0<r<1, p>0 .
$$

Since $S t^{*}$ is a compact subset of $\mathscr{A}$ we know, by arguments given in [12], that

$$
\max _{f \in S t^{*}} J\left(f^{(n)}\right)=\max _{f \in \mathfrak{\Phi} S t^{*}} J\left(f^{(n)}\right)=\max _{f \in \llbracket \mathfrak{s} S t^{*}} J\left(f^{(n)}\right)
$$

for $n=0,1,2, \cdots, 0<r<1$ if $p \geqq 1$. Theorem 2 asserts that 


$$
\text { ESSSt } \mathbb{S}^{*}=\left\{f: f(z)=\frac{y z}{(1-x z)^{2}},|x|=|y|=1\right\}
$$

and since $J\left(f^{(n)}\right)$ is constant on these functions we conclude that

$$
\frac{1}{2 \pi} \int_{0}^{2 \pi}\left|f^{(n)}\left(r e^{i \theta}\right)\right|^{p} d \theta \leqq \frac{1}{2 \pi} \int_{0}^{2 \pi}\left|k^{(n)}\left(r e^{i \theta}\right)\right|^{p} d \theta \quad(n=0,1,2, \cdots)
$$

for each function $f$ in $S t^{*}$, where

$$
0<r<1, p \geqq 1 \text {, and } k(z)=\frac{z}{(1-z)^{2}} .
$$

The inequalities (5) hold, more generally, for the class $C^{*}$. This depends on arguments given by the second author in [12] for $n=$ $1,2,3, \cdots$ and by D. R. Wilken in [20] for $n=0$. We note that the inequalities (5) contain the result $\left|a_{n}\right| \leqq n$ for the coefficients of $f$. This follows for a given $n$ by using the inequality for $p=1$ and then by letting $r \rightarrow 0$. Similar results about $L^{p}$ means hold for functions in $K^{*}$, as is easy to show (see [12] for various references about related problems).

4. General results on the extreme points of the family of functions subordinate to a given function.

THEOREM 6. Let $F$ be analytic in $\triangle$ and let $\mathscr{F}$ be the family of functions subordinate to $F$ in $\Delta$. Then the functions $f(z)=F(x z)$, $|x|=1$, belong to [S $\mathscr{S} \mathscr{F}$.

Proof. The theorem holds if $F$ is constant. Otherwise, we let $F(z)=A_{0}+A_{n} z^{n}+A_{n+1} z^{n+1}+\cdots(|z|<1)$ where $A_{n} \neq 0$. Suppose that $f \in \mathscr{F}$ and $f(z)=a_{0}+a_{1} z+a_{2} z^{2}+\cdots$. Then $a_{0}=A_{0}$, and if we write $f(z)=F(\phi(z))$ where $\phi(z)=c_{1} z+c_{2} z^{2}+\cdots$, then $a_{1}=a_{2}=\cdots=$ $a_{n-1}=0$ and $a_{n}=c_{1}^{n} A_{n}$. Since $|\phi(z)|<1$ for $|z|<1$ it follows that $\left|c_{1}\right| \leqq 1$ and $\left|c_{1}\right|=1$ only if $\phi(z)=x z$ and $|x|=1$. This implies that $\operatorname{Re} a_{n} \leqq\left|A_{n}\right|$ and that $\operatorname{Re} a_{n}=\left|A_{n}\right|$ only for functions $f(z)=F(x z)$ where $|x|=1$ (and $x$ has a suitable argument so that $x^{n} A_{n}>0$ ).

Let $\mathscr{F}_{0}$ consist of the functions $f(z)=\sum_{k=0}^{\infty} a_{k} z^{k}$ in $\mathscr{S} \mathscr{F}$ for which $\operatorname{Re} a_{n}=\left|A_{n}\right|$. Then $\mathscr{F}_{0}$ is a compact subset of $\mathscr{S} \mathscr{F}$ and thus contains an extremal element [5, p. 439], say $f_{0}$. Since $\mathscr{F}_{0}$ is an extremal subset of $\mathscr{S} \mathscr{F}$ this implies that $f_{0} \in \mathfrak{F} \mathscr{F} \mathscr{F}$. But as $\mathscr{F}$ is compact $\left[5 \mathscr{F} \mathscr{F} \subset \mathscr{F}\left[5\right.\right.$, p. 440] and so $f_{0} \in \mathscr{F}$. Therefore, $f_{0}$ must have the form $f_{0}(z)=F(x z)$ for a suitable $x$, as shown above $(|x|=1)$. This shows that there is a complex number $x_{1}$ so that $\left|x_{1}\right|=1$ and $F\left(x_{1} z\right) \in \mathbb{F} \mathscr{S}_{\mathcal{C}} \mathscr{F}$. 
We next show that if $F\left(x_{1} z\right) \in$ FSF $\mathscr{F}$ for some $x_{1},\left|x_{1}\right|=1$ then $F(x z) \in \mathbb{F} \mathscr{S} \mathscr{F}$ for every $x,|x|=1$. Suppose that $\left|x_{2}\right|=1 F\left(x_{2} z\right) \notin$ FS $\mathscr{F}$. Then, we may write $F\left(x_{2} z\right)=t f(z)+(1-t) g(z)$ where $0<t<1, f \neq$ $g$ and $f$ and $g$ belong to $\mathscr{S} \mathscr{F}$. This is the same as $F\left(x_{1} z\right)=t f\left(x_{1} / x_{2} z\right)+$ $(1-t) g\left(x_{1} / x_{2} z\right)$, which would contradict that $F\left(x_{1} z\right) \in \mathbb{F} \mathcal{S}_{\mathcal{F}} \mathscr{F}$ if we show that $f\left(x_{1} / x_{2} z\right)$ and $g\left(x_{1} / x_{2} z\right)$ belong to $\mathscr{F} \mathscr{F}$, since $f\left(x_{1} / x_{2} z\right) \neq g\left(x_{1} / x_{2} z\right)$.

We are required to show that if $|x|=1$ and $f \in \mathscr{F}_{\mathcal{F}} \mathscr{F}$ then $f(x z) \in \mathfrak{F} \mathscr{F}$. A function $f \in \mathscr{S} \mathscr{F}$ if $f$ is a limit (uniform on compact subsets of 4 ) of functions of the form $\sum_{k=1}^{n} \lambda_{k} f_{k}$, where $\lambda_{k} \geqq 0$, $\sum_{k=1}^{n} \lambda_{k}=1$ and $f_{k} \in \mathscr{F}$. Since this is the same as $f(x z)$ is the limit of functions of the form $\sum_{k=1}^{n} \lambda_{k} f_{k}(x z)$ we need only show that if $g \in \mathscr{F}$ then $g(x z) \in \mathscr{F}$. If $g \in \mathscr{F}$ we may write $g(z)=F(\phi(z))$ where $\phi$ is analytic in $\Delta,|\phi(z)|<1$ and $\phi(0)=0$. This implies that $g(x z)=$ $F(\phi(x z))$, and as $\phi(x z)$ has the same properties as $\phi$, that $g$ is subordinate to $F$. Thus, $g \in \mathscr{F}$. This completes the proof.

Theorem 6 can be proved by appealing to other unique extremal properties of the functions $F(x z)$. For example, say that $F^{\prime}(0) \neq 0$, so that for all sufficiently small $r, F$ maps $|z|<r$ one-to-one onto a convex domain. Lindelof's theorem [13, p. 22] asserts that if $\Delta_{r}=$ $\{z:|z|<r\}$ then $f\left(\Delta_{r}\right) \subset F\left(\Delta_{r}\right)$ and the boundary of $f\left(\Delta_{r}\right)$ meets the boundary of $F\left(\Delta_{r}\right)$ only if $f(z)=F(x z),|x|=1$. The convexity of $F\left(\Delta_{r}\right)$ thereby implies that $\operatorname{Re} e^{i \alpha} f(z)$ is uniquely maximized for each $z,|z|=r$, over $\mathscr{F}$. With varying $\alpha$ we get all functions $F(x z)$, $|x|=1$.

Theorem 6 may be thought of as prescribing the minimal possibility for the set $\mathfrak{F} \mathscr{S} \mathscr{F}$. This minimal situation is achieved for several examples discussed earlier. Our next theorem gives information about when the set ${ }_{5} \mathcal{S}_{\mathcal{F}}$ can be much more varied for certain functions $F$.

We need to recall some results about subordination and its relation to $H^{p}$ spaces. (For a general discussion of the theory of $H^{p}$ spaces see [3] or [9].) For a function $f$ analytic in $\Delta$ we set

$$
\mathscr{M}_{p}[f ; r]=\left\{\frac{1}{2 \pi} \int_{0}^{2 \pi}\left|f\left(\mathrm{re}^{i \theta}\right)\right|^{p} d \theta\right\}^{1 / p}
$$

$(p>0,0<r<1)$. In [11] J. E. Littlewood proved that if $f \prec F$ then

$$
\mathscr{M}_{p}[f ; r] \leqq \mathscr{M}_{p}[F ; r]
$$

for $p>0,0<r<1$. Also, $\mathscr{A}_{p}[f ; r]$ is an increasing function of $r$ $(0<r<1)[3$, p. 9]. 
The space $H^{p}(p \geqq 1)$ is defined to be the set of functions $F$ analytic in $\Delta$ for which $\mathscr{M}_{p}[F ; r]$ is a bounded function of $r$ for $0<$ $r<1$. If $F \in H^{p}$ then we set $\|F\|_{p}=\lim _{r \rightarrow 1} \mathscr{M}_{p}[F ; r]$. For such functions, $\lim _{r \rightarrow 1} F\left(\mathrm{re}^{i \theta}\right)$ exists for almost all $\theta$ and defines a function $F\left(e^{i \theta}\right)$ which we also denote by $F$. It follows that

$$
F \in L^{p}(0,2 \pi) \text { and }\|F\|_{p}=\left\{\frac{1}{2 \pi} \int_{0}^{2 \pi}\left|F\left(e^{i \theta}\right)\right|^{p} d \theta\right\}^{1 / p} .
$$

Now, if $f \prec F$ and $F \in H^{p}$ these several relations imply that $f \in H^{p}$ and $\|f\|_{p} \leqq\|F\|_{p}$. A study of these and other $H^{p}$ relations between $f$ and $F$ was presented by J. V. Ryff in [18], including the proof of Theorem $B$ stated below. We first recall the definition of an inner function $\phi$. This means that $\phi$ is analytic in $\Delta,|\phi(z)|<1$ and $\left|\phi\left(e^{i \theta}\right)\right|=1$ for almost all $\theta$.

Theorem B. (Ryff) Suppose that $f \prec F$ and $F \in H^{p}(p \geqq 1)$. A necessary and sufficient condition that $\|f\|_{p}=\|F\|_{p}$ is that $f=F(\phi)$ where $\phi$ is an inner function and $\phi(0)=0$.

THEOREM 7. Let $F$ be analytic in $\Delta$ and let $\mathscr{F}$ be the family of functions subordinate to $F$ in $\Delta$. If $F \in H^{p}$ where $1<p<\infty$ and if $\phi$ is an inner function so that $\phi(0)=0$, then $f=F(\phi) \in \mathbb{F} \mathcal{F}_{\mathcal{F}} \mathscr{F}$.

Proof. We first show the following general statement: If $F \in H^{p}$ and $p \geqq 1$ then $\mathfrak{F} \mathscr{F} \subset H^{p}$. Suppose that $f \in \mathfrak{S} \mathscr{F}$. Then $f$ can be uniformly approximated on compact subsets of $\Delta$ by functions of the form $\sum_{k=1}^{n} \lambda_{k} f_{k}$ where $\lambda_{k} \geqq 0, \sum_{k=1}^{n} \lambda_{k}=1$ and $f_{k} \in \mathscr{F}$. Since $f_{k} \prec F$ Littlewood's inequality implies that $\mathscr{N}_{p}\left[f_{k} ; r\right] \leqq \mathscr{N}_{p}[F ; r]$ for each $r(0<r<1)(k=1,2, \cdots, n)$. We may use the Minkowski inequality since $p \geqq 1$ and this shows that

$$
\mathscr{M}_{p}\left[\sum_{k=1}^{n} \lambda_{k} f_{k} ; r\right] \leqq \sum_{k=1}^{n} \lambda_{k} \mathscr{M}_{p}\left[f_{k} ; r\right] \leqq \mathscr{M}_{p}[F ; r] .
$$

This implies that $\mathscr{C}_{p}[f ; r] \leqq \mathscr{C}_{p}[F ; r]$ and, therefore, $f \in H^{p}$ and $\|f\|_{p} \leqq\|F\|_{p}$. Also $\lim _{r \rightarrow 1} f\left(\mathrm{re}^{i \theta}\right)=f\left(e^{i \theta}\right)$ exists for almost all $\theta$ and

$$
\|f\|_{p}=\left\{\frac{1}{2 \pi} \int_{0}^{2 \pi}\left|f\left(e^{i \theta}\right)\right|^{p} d \theta\right\}^{1 / p} .
$$

Now, suppose that $F \in H^{p}$ and $f=F(\phi)$ where $\phi$ is an inner function and $\phi(0)=0$. Also, suppose that $f(z)=t g(z)+(1-t) h(z)$ $(z \in \Delta)$ where $0<t<1$ and $g, h$ belong to $\mathscr{S} \mathscr{F}$. By the argument in the previous paragraph the functions $g\left(e^{i \theta}\right)=\lim _{r \rightarrow 1} g\left(\mathrm{re}^{i \theta}\right)$ and $h\left(e^{i \theta}\right)=$ $\lim _{r \rightarrow 1} h\left(\mathrm{re}^{i \theta}\right)$ are well-defined (for almost all $\theta$ ). They belong to 


$$
L^{p}(0,2 \pi),\|g\|_{p}=\left(\frac{1}{2 \pi} \int_{0}^{2 \pi}\left|g\left(e^{i \theta}\right)\right|^{p} d \theta\right)^{1 / p}
$$

and

$$
\|h\|_{p}=\left(\frac{1}{2 \pi} \int_{0}^{2 \pi}\left|h\left(e^{i \theta}\right)\right|^{p} d \theta\right)^{1 / p}
$$

Also, $f\left(e^{i \theta}\right)=t g\left(e^{i \theta}\right)+(1-t) h\left(e^{i \theta}\right)$ (for almost all $\theta$ ). By the Minkowski inequality this implies that $\|f\|_{p} \leqq t\|g\|_{p}+(1-t)\|h\|_{p} \leqq t\|F\|_{p}+$ $(1-t)\|F\|_{p}=\|F\|_{p}$. Because of Theorem B, the inequalities here each must be an equality. But when $p>1$ equality in the Minkowski inequality is possible only when the two functions are effectively multiples of each other. Therefore, there is a number $\alpha \neq 0$ so that $\operatorname{tg}\left(e^{i \theta}\right)=$ $\alpha(1-t) h\left(e^{i \theta}\right)$ for almost all $\theta$. When two functions in $H^{p}$ have boundary values that are equal on a set of positive measure they are equal as functions in $\Delta$. Thus, $t g(z)=\alpha(1-t) h(z)$ for each $z$ in $\Delta$. Since $g(0)=h(0)=F(0)$ this implies that $t F(0)=\alpha(1-t) F(0)$. We may assume that $F(0) \neq 0$, otherwise we would first prove the theorem, say, with $F(z)+1$ replacing $F(z) . \quad F(0) \neq 0$ implies that $t=\alpha(1-t)$ and so $t g=\alpha(1-t) h$ is equivalent to $\alpha(1-t) g=\alpha(1-t) h$, or $g=h$. This proves that $f \in \mathbb{F} \mathscr{S}_{\mathcal{C}} \mathscr{F}$ if $p>1$.

REMARKs 1. The result of Theorem 7 contrasts sharply with the earlier situations. For example, we now know something about the diversity of $\mathbb{F} \mathscr{S}_{\mathcal{F}} \mathscr{F}$ where $\mathscr{F}$ consists of the functions subordinate to $F(z)=G^{q}(z)$ where $G$ is a linear fractional transformation and $q<$ 1 because this implies that $F \in H^{p}$ for some $(1<p<\infty)$.

2. There are other ways to show that such a family $\mathscr{F}$ has extreme points besides the functions $F(x z),|x|=1$, without obtaining such descriptive information as that given by Theorem 7 . A good example of this is provided by the result proved by A. W. Goodman in [7]. He shows that if the class of functions subordinate to a univalent function $F$ is the same as the set of functions given by $f(z)=\int_{|x|=1} F(x z) d \mu(x)$, where $\mu$ varies over the probability measures on $|x|=1$, then $F(\Delta)$ is a half-plane. The converse is, of course, equivalent to Herglotz's formula.

For example, let $\mathscr{F}$ denote the family of functions subordinate to $F(z)=1 /(1-z)^{p}$ in $\Delta$ where $0<p<1$. If $5 \mathcal{S} \mathscr{F}$ contained only the functions $F(x z),|x|=1$, then by the Krein-Milman theorem we would conclude that $\mathscr{S} \mathscr{F}$ consists of the functions $f(z)=\int_{|x|=1} F(x z)$ $d \mu(x)$ ( $\mu$ varying over the probability measures.) But, as is easy to 
verify, $F(z)$ is univalent and convex since $0<p<1$ and so the functions given by these integrals would be all functions subordinate to $F$, contrary to Goodman's result.

One can also show that the functions $F(x z),|x|=1$, are not the only extreme points by showing that none of these functions solves a specific linear problem. For example, with $F(z)=1 /(1-z)^{p}=1+$ $A_{1} z+A_{2} z^{2}+\cdots$, we see that $A_{2}=p(p+1) / 2$ and so if $F(x z)=1+$ $A_{1}(x) z+A_{2}(x) z^{2}+\cdots,|x|=1$, then $\left|A_{2}(x)\right|=p(p+1) / 2$. But we know that $\max \left|a_{2}\right|=p>p(p+1) / 2$, where $f(z)=1+a_{1} z+a_{2} z^{2}+\cdots$ varies over functions subordinate to $F$.

The type of situation just described also occurs if one of the coefficients (after $A_{1}$ ) of $F$ vanishes. Similarly, if $F(z)=\sum_{n=0}^{\infty} A_{n} z^{n}$, $A_{1} \neq 0$ and $A_{n} \rightarrow 0$ then there are extreme points other than $F(x z)$, $|x|=1$, as such functions are not extremal for the problem $\max \left|a_{n}\right|$ if $n$ is sufficiently large. This follows merely by noting that the functions $f(z)=F\left(z^{n}\right)$ are subordinate to $F$. The situation $A_{n} \rightarrow 0$ occurs, for example, if $F$ is univalent and convex in $\Delta$ and does not map onto a half-plane. More generally, $F$ need only be subordinate to some such function and $F^{\prime}(0) \neq 0$. To see this observe that such a function belongs to $H^{1}$ due to Littlewood's inequality and the fact that a convex, univalent function other than a half-plane mapping is in $H^{1}$ (and even in $H^{p}$ for some $p>1$ ). But it always follows that $A_{n} \rightarrow 0$ if $F \in H^{1}$. A geometric realization of this situation occurs whenever the range of $F$ has at least two distinct lines of support.

3. The converse of Theorem 7 is not generally true; for certain functions $F$ in $H^{p}$ there can be extreme points not of the form $F(\phi)$ where $\phi$ is an inner function. This is already the case when $F(z)=$ $z$ [see 9, p. 138]. We thank L. Brickman for pointing out the following example in which extreme points can be geometrically realized. Let the analytic function $F$ be univalent in $\Delta$ so that $F(\Delta)$ is convex and some arc $\Gamma$ on the boundary of $F(\Delta)$ does not contain a line segment. Then each analytic function $f$ (say, univalent in $\Delta$ ) so that $f(0)=F(0), f(\Delta) \subset F(\Delta)$ and [closure of $f(\Delta)] \cap$ [closure of $F(\Delta)] \supset \Gamma$ is extreme in the class of functions subordinate to $F$. To see this consider writing $f(z)=t g(z)+(1-t) h(z)(z \in \Delta)$ where $0<t<1, g \in \mathscr{S} \mathscr{F}$ and $h \in \mathscr{F} \mathscr{F}$. Since $F(\Delta)$ is convex $\mathscr{F} \mathscr{F}=\mathscr{F}$ and so $g$ and $h$ belong to $H^{1}$. Therefore, $g$ and $h$ have radial limits almost everywhere. We now see that by taking radial limits associated with points $e^{i \theta}$ so that $f\left(e^{i \theta}\right) \in \Gamma$ that the relation $f(z)=t g(z)+(1-t) h(z)$ could only be possible if $g(z)=h(z)=f(z)$ on some set on $|z|=1$ of positive measure. But then we would have $g=h$ in $\Delta$. 


\section{REFERENCES}

1. D. A. Brannan, J. G. Clunie, and W. E. Kirwan, On the coefficient problem for functions of bounded boundary rotation, Ann. Acad. Sci. Fenn. Ser. A1 Math. Phys. 523 (1973), $18 \mathrm{pp}$.

2. L. Brickman, T. H. MacGregor, and D. R. Wilken, Convex hulls of some classical families of univalent functions, Trans. Amer. Math. Soc., 156 (1971), 91-107.

3. P. L. Duren, Theory of $H^{p}$ Spaces, Academic Press, New York, 1970.

4. P. L. Duren and G. E. Schober, On a class of Schlicht functions, Michigan Math. J., 18 (1971), 353-356.

5. N. Dunford and J. T. Schwartz, Linear Operators, I: General theory, Pure and applied Math., vol. 7, Interscience, New York, 1958.

6. O. Dvorak, Ofunkcich prostych, Casopis Pest. Mat., 63 (1934), 9-16.

7. A. W. Goodman, Analytic functions that take values in a convex region, Proc. Amer. Math. Soc., 14 (1963), 60-64.

8. E. Hille, Analytic Function Theory, vol. 2, Ginn, New York, 1962.

9. K. Hoffman, Banach Spaces of Analytic Functions, Prentice-Hall, Englewood Cliffs, 1962.

10. W. Kaplan, Close-to-convex Schlicht functions, Michigan Math. J., 1 (1952), 169-185. 11. J. E. Littlewood, On inequalities in the theory of functions, Proc. London Math. Soc., (2), 23 (1925), 481-519.

12. T. H. MacGregor, Applications of extreme-point theory to univalent functions, Michigan Math. J., 19 (1972), 361-376.

13. Z. Nehari, Conformal Mappings, McGraw-Hill, New York, 1952.

14. P. Porcelli, Linear Spaces of Analytic Functions, Rand McNally, Chicago, 1966.

15. M. S. Robertson, The generalized Bieberbach conjecture for subordinate functions, Michigan Math. J., 12 (1965), 421-429.

16. W. Rogosinski, Uber positive harmonische Entwicklungen und typisch-reelle Potenzreiken, Math. Zeit., 35 (1932), 93-121.

17. - On the coefficients of subordinate functions, Proc. London Math. Soc., (2) 48 (1943), 48-82.

18. J. V. Ryff, Subordinate $H^{p}$ functions, Duke Math. J., 33 (1966), 347-354.

19. A. E. Taylor, Introduction to Functional Analysis, Wiley, New York, 1958.

20. D. R. Wilken, The integral means of close-to-convex functions, Michigan Math. J., 19 (1972), 377-379.

Received September 28, 1972 and in revised form October 15, 1973. Partially supported by the National Science Foundation Grant GP-19709.

State University of New York at Albany 


\section{PACIFIC JOURNAL OF MATHEMATICS}

\section{EDITORS}

RICHARD ARENS (Managing Editor)

University of California

Los Angeles, California 90024

\section{R. A. Beaumont \\ University of Washington \\ Seattle, Washington 98105}

\section{J. DugundjI*}

Department of Mathematics University of Southern California Los Angeles, California 90007

D. Gilbarg and J. Milgram

Stanford University

Stanford, California 94305

\section{ASSOCIATE EDITORS}
E. F. BeCKenBaCH
B. H. NeumanN
F. WOLF
K. YoSHIDA

\section{SUPPORTING INSTITUTIONS}

\author{
UNIVERSITY OF BRITISH COLUMBIA \\ CALIFORNIA INSTITUTE OF TECHNOLOGY \\ UNIVERSITY OF CALIFORNIA \\ MONTANA STATE UNIVERSITY \\ UNIVERSITY OF NEVADA \\ NEW MEXICO STATE UNIVERSITY \\ OREGON STATE UNIVERSITY \\ UNIVERSITY OF OREGON \\ OSAKA UNIVERSITY
}

\author{
UNIVERSITY OF SOUTHERN CALIFORNIA \\ STANFORD UNIVERSITY \\ UNIVERSITY OF TOKYO \\ UNIVERSITY OF UTAH \\ WASHINGTON STATE UNIVERSITY \\ UNIVERSITY OF WASHINGTON

$* * *$
$*$
AMERICAN MATHEMATICAL SOCIETY
NAVAL WEAPONS CENTER

The Supporting Institutions listed above contribute to the cost of publication of this Journal, but they are not owners or publishers and have no responsibility for its content or policies.

Mathematical papers intended for publication in the Pacific Journal of Mathematics should be in typed form or offset-reproduced, (not dittoed), double spaced with large margins. Underline Greek letters in red, German in green, and script in blue. The first paragraph or two must be capable of being used separately as a synopsis of the entire paper. Items of the bibliography should not be cited there unless absolutely necessary, in which case they must be identified by author and Journal, rather than by item number. Manuscripts, in duplicate if possible, may be sent to any one of the four editors. Please classify according to the scheme of Math. Rev. Index to Vol. 39. All other communications to the editors should be addressed to the managing editor, or Elaine Barth, University of California, Los Angeles, California, 90024.

100 reprints are provided free for each article, only if page charges have been substantially paid. Additional copies may be obtained at cost in multiples of 50 .

The Pacific Journal of Mathematics is issued monthly as of January 1966. Regular subscription rate: $\$ 60.00$ a year (6 Vols., 12 issues). Special rate: $\$ 30.00$ a year to individual members of supporting institutions.

Subscriptions, orders for back numbers, and changes of address should be sent to Pacific Journal of Mathematics, 103 Highland Boulevard, Berkeley, California, 94708.

PUBLISHED BY PACIFIC JOURNAL OF MATHEMATICS, A NON-PROFIT CORPORATION

Printed at Kokusai Bunken Insatsusha (International Academic Printing Co., Ltd.), 270, 3-chome Totsuka-cho, Shinjuku-ku, Tokyo 160, Japan

* C. R. DePrima California Institute of Technology, Pasadena, CA 91109, will replace J. Dugundji until August 1974. 


\section{Pacific Journal of Mathematics}

Vol. 50, No. $2 \quad$ October, 1974

Mustafa Agah Akcoglu, John Philip Huneke and Hermann Rost, A counter example to the Blum Hanson theorem in general spaces .............

Huzihiro Araki, Some properties of modular conjugation operator of von

Neumann algebras and a non-commutative Radon-Nikodym theorem

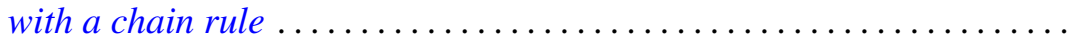

E. F. Beckenbach, Fook H. Eng and Richard Edward Tafel, Global properties of rational and logarithmico-rational minimal surfaces .....

David W. Boyd, A new class of infinite sphere packings ............. 383

K. G. Choo, Whitehead Groups of twisted free associative algebras ........

Charles Kam-Tai Chui and Milton N. Parnes, Limit sets of power series

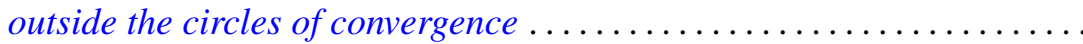

Allan Clark and John Harwood Ewing, The realization of polynomial

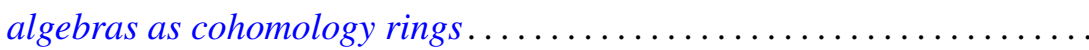

Dennis Garbanati, Classes of circulants over the p-adic and rational

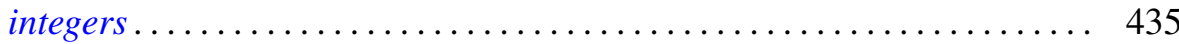

Arjun K. Gupta, On a "square" functional equation ................... 449

David James Hallenbeck and Thomas Harold MacGregor, Subordination and extreme-point theory ............................. 455

Douglas Harris, The local compactness of $v X \ldots \ldots . . . . . . . . . . . .4469$

William Emery Haver, Monotone mappings of a two-disk onto itself which fix the disk's boundary can be canonically approximated by homeomorphisms .................................. 477

Norman Peter Herzberg, On a problem of Hurwitz .................. 485

Chin-Shui Hsu, A class of Abelian groups closed under direct limits and subgroups formation ............................... 495

Bjarni Jónsson and Thomas Paul Whaley, Congruence relations and multiplicity types of algebras.....................

Lowell Duane Loveland, Vertically countable spheres and their wild sets.

Nimrod Megiddo, Kernels of compound games with simple components ....

Russell L. Merris, An identity for matrix functions ........ . .

E. O. Milton, Fourier transforms of odd and even tempered distributions ...

Dix Hayes Pettey, One-one-mappings onto locally connected generalized continua

Mark Bernard Ramras, Orders with finite global dimension

Doron Ravdin, Various types of local homogeneity. .

George Michael Reed, On metrizability of complete Moore spaces ...

Charles Small, Normal bases for quadratic extensions ..

Philip C. Tonne, Polynomials and Hausdorff matrices.... . . 